\title{
Controllability, Observability in Networked Control
}

\author{
Stefano Longo* Guido Herrmann* Phil Barber ** \\ * Department of Mechanical Engineering, Faculty of Engineering, The \\ University of Bristol, Queen's Building, University Walk, Bristol, BS8 1TR, \\ UK, (Fax: +441179294423; tel. S. Longo: +441173317371; tel. G. \\ Hermann: +441179288100; e-mails: $\{$ s.longo, \\ g.herrmann\}@bristol.ac.uk). \\ ** Jaguar and Land Rover Research, W/2/021 Engineering Centre, Abbey \\ Road, Whitley, Coventry, CV $4 L F, U K$.
}

\begin{abstract}
We reconsider and advance the analysis of structural properties (controllability and observability) of a class of linear Networked Control Systems (NCSs). We model the NCS as a periodic system with limited communication where the non updated signals can either be held constant (the zero-order-hold case) or reset to zero. Periodicity is dealt using the lifting technique. We prove that a communication sequence that avoids particularly defined pathological sampling rates and updates each actuator signal only once is sufficient to preserve controllability (and observability for the dual problem of sensor scheduling). These sequences can be shorter than previously established and we set a tight lower bound to them.
\end{abstract}

\section{INTRODUCTION}

In a Networked Control System (NCS), sensors, actuators and controllers are spatially distributed and interconnected via a shared communication medium. More generally, in a NCS, all control loops are closed through a real-time network. NCS increase modularity, flexibility and allow quick and easy maintenance at low cost. They are essential to automotive industry (see Leen and Heffernan [2002]), avionic systems (see Gwaltney and Briscoe [2006]), robots (see Oda et al. [2001], Göktas et al. [1997]) and automated manufacturing systems (see Lian et al. [2000]) to reduce hardwiring and costs of installation and implementation. For example, a typical modern car has a highspeed Controller Area Network (CAN) in front of the firewall for engine, transmission and traction control and a low-speed one for locks, windows and other devices (see Walsh and Ye [2001]).

The presence of the network inevitably introduces new challenges for the control engineering community that lie in the intersection of control and communication theory. Since information exchange has to be somehow time multiplexed, the inevitable effect is the so called network-induced delay. Networkinduced delays can be infinitely long (packet dropout) and non-deterministic (queues and bandwidth contention). Contrary to data networks, data, in control networks, are continuously transmitted because they have to meet critical time requirements. Package loss, delays and non-determinism seriously affect the stability and performance of the networked system and may not be acceptable for safety-related control systems.

There are two main fundamentally different communication protocols: contention-free and contention-based. The focus in this paper are contention-free protocols where the sequence of the control signal is solely dependent on the progression in time and therefore allows pre-planning of a particular periodic communication sequence at the design stage. Contention-free architectures increase determinism and are more suited for predictable hard real-time applications (see Kopetz [1991]).

Structural properties (reachability and observability) and stabilization of linear NCS are discussed in Zhang and HristuVarsakelis [2006], Ionete and Çela [2006] but only for the less complex setting where no zero-order-hold $(\mathrm{ZOH})$ element is placed between controller and plant. In Hristu-Varsakelis [2007, 2008], the structural analysis is extended to include a $\mathrm{ZOH}$ strategy and new results on the period of the communication sequence are presented. Zhang and Hristu-Varsakelis [2006], Ionete and Çela [2006], Hristu-Varsakelis [2007, 2008] proved that communication sequences that preserve reachability and observability exist, have an upper bound on their period and can be easily found by a simple iterative algorithm. The stabilizability and detectability properties of a multirate sampled-data system are discussed in Colaneri et al. [1992]. Multirate systems can be considered as special cases of NCSs with communication schedule.

In this paper, we reconsider and advance the analysis of structural properties (controllability and observability) of a class of NCS. We consider a system where the actuator nodes share a common bus and only a limited number of actuator signals can be updated at every time tick. We mainly focus on the more realistic $\mathrm{ZOH}$ case where all the non-updated control signals will remain the same until updated. The main question we are trying to answer is: given a continuous-time controllable plant model, what are the properties of an actuator communication scheduling sequence that preserves controllability when the system is implemented as a NCS? Once these properties are defined, then it is easy to find a scheduler with a fixed, periodic communication sequence that preserves controllability. 
The problem of finding a sensor communication scheduling sequence that preserves observability is the dual problem and the equivalent result is discussed.

The approach is extended to the case where no $\mathrm{ZOH}$ element is placed between controller and actuators and also the sensor signals are scheduled. It will be shown that a sequence that preserves controllability (and observability for the dual problem) can be 'minimal' in length and therefore shorter than previously established by Zhang and Hristu-Varsakelis [2006], Ionete and Çela [2006], Hristu-Varsakelis [2007, 2008].

\section{NCS MODEL}

In this section, we introduce the theoretical framework of limited communication for control. The framework follows the one of Rehbinder and Sanfridson [2004] and extends it to the multi-channel case of Ben Gaid et al. [2006].

\subsection{Scheduling problem}

Consider the NCS shown in Fig. 1. The plant actuators are

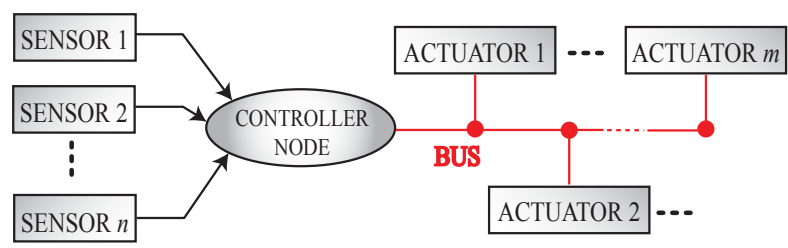

Fig. 1. Architecture of a NCS with limited communication: the actuators communicate through a shared bus where a reduced number of control signals can be updated at any time tick.

spatially distributed and the limited communication medium used for the actuator signals is represented by a shared bus. A scheduler acts in the form of switches which are nodes between the end-points and the bus. For simplicity, sensor signals are not subject to bus communication constraints; this is the dual problem that can be stated in a similar way. Only a limited number of actuators (often only one) can be controlled and we assume that the actuator inputs latch so that when the switch contact is opened the actuator holds its signal value $(\mathrm{ZOH})$. We assume that the spatially distributed plant is a linear timeinvariant system described by

$$
\dot{x}(t)=A x(t)+B u(t), \quad x(0)=x_{0},
$$

where $x(t) \in \mathbb{R}^{n}, u(t) \in \mathbb{R}^{m},(A, B)$ is a controllable pair and $\operatorname{rank}(B)=m$. An additional important assumption here is that all $m$ control signals are needed to retain the plant $(A, B)$ controllable. The system described by (1) may be a decoupled system communicating the control signals over a single bus. The control input $u(t)$ is a discrete input signal created by a $\mathrm{ZOH}$ element for a constant sampling period $h$ :

$$
u(t)=u(j h)=\text { const. for } t \in[j h,(j+1) h) .
$$

The model in (1) has to be sampled with a periodic sampling interval $h$ giving the sampled-data time-invariant system for constant input $u$ (defined in (2)):

$$
x(j h+h)=\Phi x(j h)+\Gamma u(j h), \quad x(0)=x_{0},
$$

where

$$
\begin{aligned}
& \Phi=\check{\Phi}(h), \quad \Gamma=\check{\Gamma}(h), \\
& \check{\Phi}(\tau)=e^{A \tau}, \quad \check{\Gamma}(\tau)=\int_{0}^{\tau} e^{A s} d s B,
\end{aligned}
$$

and $j$ is the sampling instant.

Remark 1. If the sampling frequency is non-pathological (i.e. $A$ does not have two eigenvalues with equal real parts and imaginary part that differ by an integral multiple of $\frac{2 \pi}{h}$, [Chen and Francis, 1995, Theorem 3.2.1]), then

$$
\begin{aligned}
(A, B) \text { controllable } & \Rightarrow(\Phi, \Gamma) \text { controllable } \\
(C, A) \text { observable } & \Rightarrow(C, \Phi) \text { observable, }
\end{aligned}
$$

where $C$ is an output matrix. This is a sufficient condition for preservation of controllability and observability after discretization (necessary and sufficient conditions can be found in Kimura [1990]).

For simplicity, we will omit the sampling period $h$ from now on.

The next step is to obtain a model for the scheduler that emulates the limited communication channel (see Rehbinder and Sanfridson [2004], Ben Gaid et al. [2006]). The scheduler acts like selector switches with as many positions as the number of actuators $m$ to be controlled. We need to define the concept of communication sequence (see Brockett [1995]) and sequence of scheduling matrices. From a practical viewpoint, it is reasonable to assume that sequences are periodic.

Definition 1. If $m$ is the number of actuators, $m_{\max }$ the maximum number of control signals that can be transmitted at any time tick $t=i h$ and $m_{r}(k), 1 \leq m_{r}(k) \leq$ $m_{\max } \leq m$, for all $k$ is the reduced number of actuators controlled at time $k$, a $p$-periodic communication sequence $\sigma=\{\sigma(0), \sigma(1), \ldots, \sigma(p-1)\}$ is an ordered list of $p$ vectors $\sigma(k)=\left[\sigma_{1}(k) \sigma_{2}(k) \ldots \sigma_{m_{r}(k)}(k)\right]^{T}$ where $\sigma_{i}(k) \in \mathbb{N}$, $1 \leq \sigma_{i}(k) \leq m$, is the index of the actuator for which control communication is enabled at time tick $k$.

Definition 2. The sequence of binary scheduling matrices is $S=\{S(0), S(1), \ldots, S(p-1)\}$ where $S(k):=\left(s_{r, c}(k)\right)_{m \times m}$ and $s_{r, c}(k)$ are the elements of $S(k)$ in the $r^{t h}$ row and $c^{t h}$ column. The elements $s_{r, c}(k)$ satisfy:

$$
\begin{aligned}
& s_{r, c}(k)= \begin{cases}1 & \text { if } r=c=\sigma_{i}(k) \\
0 & \text { otherwise }\end{cases} \\
& \forall r, c=1,2, \ldots m ; i=1,2, \ldots, m_{r}(k) .
\end{aligned}
$$

We also define $\bar{S}=\{\bar{S}(0), \bar{S}(1), \ldots, \bar{S}(p-1)\}$, where $\bar{S}(k):=$ $\left(\bar{s}_{r, c}(k)\right)_{m \times m}$, as

$$
\bar{S}(k)=I-S(k)
$$

and $I$ is the identity matrix.

Note that $S(k)=S(k)^{T}$ and $\bar{S}(k)=\bar{S}(k)^{T}$ have a 1 or a 0 in the diagonal and zeros elsewhere and $\sum_{i=1}^{m} s_{i, i}(k)=m_{r}(k)$, $\sum_{i=1}^{m} \bar{s}_{i, i}(k)=m-m_{r}(k)$. Any permutation of the elements of $\sigma(k)$ will produce the same $S(k)$.

Having defined the $p$-periodic communication sequence and the respective scheduling matrices, the scheduler can be represented by

$$
\begin{aligned}
& v(j)=\bar{S}(k) v(j-1)+S(k) \hat{u}(j), \quad v(0)=u_{0}, \\
& k=\bmod (j, p)
\end{aligned}
$$


where $v(\cdot)$ is the limited control vector, $\hat{u}(\cdot)$ is the full control vector and $\bmod (\cdot, \cdot)$ is the modulo operator. The state matrix $S(k)$ is time-varying and $p$-periodic according to the scheduling policy. The scheduler dynamics in (9) can be understood as follows: the signal vector $v(j)$ has all its entries equal to $v(j-$ 1) apart from some entries (given by the elements of $\sigma(k)$ ) which will be equal to the ones of $\hat{u}(j)$ (the updated full control signal).

The plant model, including the limited communication can now be modified as

$$
\begin{aligned}
x(j+1) & =\Phi x(j)+\Gamma v(j) \\
& =\Phi x(j)+\Gamma(\bar{S}(k) v(j-1)+S(k) \hat{u}(j)) .
\end{aligned}
$$

We let $j=p l+k$ and merge the two system equations (11) and (9) to form the augmented system

$$
\begin{aligned}
& \hat{x}(p l+k+1)=\hat{\Phi}(k) \hat{x}(p l+k)+\hat{\Gamma}(k) \hat{u}(p l+k), \\
& \hat{x}(0)=\left[\begin{array}{ll}
x_{0}^{T} & v_{0}^{T}
\end{array}\right]^{T} .
\end{aligned}
$$

where

$$
\begin{aligned}
& \hat{x}(p l+k)=\left[\begin{array}{c}
x(p l+k) \\
v(p l+k-1)
\end{array}\right], \\
& \hat{\Phi}(k)=\left[\begin{array}{cc}
\Phi & \Gamma \bar{S}(k) \\
0 & \bar{S}(k)
\end{array}\right], \quad \hat{\Gamma}(k)=\left[\begin{array}{c}
\Gamma S(k) \\
S(k)
\end{array}\right] .
\end{aligned}
$$

The dynamics of the periodic scheduler will inevitably introduce a periodic augmented model.

\subsection{Eliminate periodicity via lifting}

To eliminate the time dependance in $k$ of the weighting matrices, the lifting technique can be used since $S(k)$ is periodic in $p$. This is described in Chen and Francis [1995] and used in Rehbinder and Sanfridson [2004], Ben Gaid et al. [2006]. The aim here is to create a higher dimensional system in order to eliminate the dependance on $k$. The lifted system from (12) is

$$
\bar{x}(p l+1)=\bar{\Phi} \hat{x}(p l)+\bar{\Gamma} \bar{u}(p l)
$$

where

$$
\begin{aligned}
& \bar{x}(p l)=\left[\hat{x}(p l)^{T} \hat{x}(p l+1)^{T} \ldots \hat{x}(p l+p-1)^{T}\right]^{T}, \\
& \bar{u}(p l)=\left[\hat{u}(p l)^{T} \hat{u}(p l+1)^{T} \cdots \hat{u}(p l+p-1)^{T}\right]^{T},
\end{aligned}
$$

and

$$
\begin{aligned}
& \bar{\Phi}=\left[\begin{array}{c}
\hat{\Phi}(0) \\
\hat{\Phi}(1) \hat{\Phi}(0) \\
\vdots \\
\hat{\Phi}(p-1) \hat{\Phi}(p-2) \cdots \hat{\Phi}(0)
\end{array}\right], \\
& \bar{\Gamma}=\left[\begin{array}{cccc}
\hat{\Gamma}(0) & 0 & \ldots & 0 \\
\hat{\Phi}(1) \hat{\Gamma}(0) & \hat{\Gamma}(1) & \ldots & 0 \\
\hat{\Phi}(2) \hat{\Phi}(1) \hat{\Gamma}(0) & \hat{\Phi}(2) \hat{\Gamma}(1) & \ldots & 0 \\
\vdots & \vdots & & \vdots \\
\hat{\Phi}(p-1) \hat{\Phi}(p-2) \ldots \hat{\Gamma}(0) & \ldots & & \hat{\Gamma}(p-1)
\end{array}\right] .
\end{aligned}
$$

The expression in (14) is in fact a set of equations of lifted systems described by

$$
\hat{x}(p l+k)=\bar{\Phi}_{k} \hat{x}(p l)+\bar{\Gamma}_{k} \bar{u}(p l)
$$

where the subscript $k$ indicates the $k^{t h}$ matrix row of $\bar{\Phi}$ and $\bar{\Gamma}$. It follows that the augmented lifted plant equation is given by the last row of the matrices in (16), therefore

$$
\hat{x}(p l+p)=\bar{\Phi}_{p} \hat{x}(p l)+\bar{\Gamma}_{p} \bar{u}(p l), \quad \hat{x}(0)=\left[\begin{array}{ll}
x_{0}^{T} & v_{0}^{T}
\end{array}\right]^{T}
$$

where

$$
\begin{aligned}
\bar{\Phi}_{p} & =\prod_{j=1}^{p} \hat{\Phi}(p-j), \\
\bar{\Gamma}_{p} & =\left[\begin{array}{lll}
G(0) G(1) \cdots G(p-1) & \cdots
\end{array},\right.
\end{aligned}
$$

and ${ }^{1}$

$$
\begin{aligned}
& G(k)=\left(\prod_{j=1}^{p-k-1} \hat{\Phi}(p-j)\right) \hat{\Gamma}(k), \\
& k=0,1, \ldots, p-1 .
\end{aligned}
$$

\subsection{Controllability}

At this point, we can state the conditions of a given communication sequence for the preservation of controllability of the lifted augmented system (18). A sufficient condition will be presented for which the following definitions are needed first.

Definition 3. The matrices $S(k)$ and $\bar{S}(k)$ can be decomposed into the matrices $Z^{(i)}(k):=\left(z_{r, c}^{(i)}(k)\right)_{m \times m}$ and $\bar{Z}^{(i)}(k):=$ $\left(\bar{z}_{r, c}^{(i)}(k)\right)_{m \times m}$ respectively where

$$
\begin{aligned}
& z_{r, c}^{(i)}(k)=\left\{\begin{array}{ll}
s_{r, c}(k) & \text { if } r=c=i \\
0 & \text { elsewhere }
\end{array},\right. \\
& \bar{z}_{r, c}^{(i)}(k)=\left\{\begin{array}{ll}
\bar{s}_{r, c}(k) & \text { if } r=c=i \\
0 & \text { elsewhere }
\end{array},\right. \\
& \forall r, c, i=1,2, \ldots, m .
\end{aligned}
$$

Note that $\sum_{i=1}^{m_{r}(k)} Z^{\left(\sigma_{i}(k)\right)}(k)=\sum_{i=1}^{m} Z^{(i)}(k)=S(k)$ and $\sum_{i=1}^{m} \bar{Z}^{(i)}(k)=\bar{S}(k)$.

Definition 4. Given a sequence $\sigma$ (Definition 1 ), let $k_{f}^{(i)}$ be the sequence indices where actuator $i$ appears for the last time in the sequence i.e. $\left\{n: n=1,2, \ldots, p-k_{f}^{(i)} ; \sigma_{j}\left(k_{f}^{(i)}\right)=\right.$ $\left.\sigma_{l}\left(k_{f}^{(i)}+n\right), \forall j, l\right\}=\varnothing$ or $\{n: n=1,2, \ldots, p-$ $\left.k_{f}^{(i)} ; s_{i, i}\left(k_{f}^{(i)}\right)=s_{i, i}\left(k_{f}^{(i)}+n\right)=1, \forall i\right\}=\varnothing$.

Remark 2. From Definition 4 , it follows that if

$$
\operatorname{rank}\left(\sum_{k=0}^{p-1} S(k)\right)=m,
$$

there will be $m$ (not necessarily distinct) $k_{f}^{(i)}$ associated with $m$ distinct matrices $Z^{(i)}\left(k_{f}^{(i)}\right)$ (i.e. $\operatorname{rank}\left(\sum_{i=1}^{m} Z^{(i)}\left(k_{f}^{(i)}\right)\right)=$ $m)$.

Definition 5. If $\operatorname{rank}\left(\sum_{k=0}^{p-1} S(k)\right)=m$, then

$$
\begin{aligned}
& \bar{q}^{(i)}=\min _{j \in\left\{j: \prod_{l=0}^{j-1} \bar{Z}^{(i)}(l)=0\right\}} j, \\
& \hat{q}^{(i)}(k)=\min \left\{\max \{k-1,0\}, \bar{q}^{(i)}\right\} .
\end{aligned}
$$

\footnotetext{
1 Note that, in this equation, when $k=p-1$, the product is undefined. For this reason, to allow for this short notation, we need to define in the context of our paper $\prod_{j=1}^{i} f(j)=1$ for $i<j$.
} 
Theorem 1. Suppose that the pair $(\Phi, \Gamma)$ (4) is controllable, $\Phi$ is invertible and $\operatorname{rank}(\Gamma)=m$. Moreover, all $m$ actuators are required for controllability of the plant, i.e. $\left(\Phi, \Gamma_{r}\right)$ is not controllable where $\Gamma_{r}$ is an input distribution matrix covering only part of the input range of $\Gamma\left(\operatorname{rank}\left(\Gamma_{r}\right)<m\right.$ and $\left.\operatorname{rank}\left(\left[\Gamma_{r} \Gamma\right]\right)=m\right)$. Under these conditions the following can be stated:

(i) The pair $\left(\bar{\Phi}_{p}, \bar{\Gamma}_{p}\right)$ (the lifted augmented system) is controllable if

$$
\begin{gathered}
\operatorname{rank}\left(\sum_{k=0}^{p-1} S(k)\right)=m, \\
\lambda \neq \exp \left(\frac{2 \pi n \sqrt{-1}}{p-k_{f}^{(i)}+\hat{q}\left(k_{f}^{(i)}\right)}\right), \quad \forall i=1,2, \ldots, m, \\
n=1,2, \ldots, p-k_{f}^{(i)}+\hat{q}\left(k_{f}^{(i)}\right)-1,
\end{gathered}
$$

for an arbitrary eigenvalue $\lambda$ of $\Phi$ and ${ }^{2}$ assuming that for any pair of eigenvalues, $\left(\lambda_{1}, \lambda_{2}\right)$ of $\Phi$, the following holds:

$$
\lambda_{1} \neq \lambda_{2} \exp \left(\frac{2 \pi l \sqrt{-1}}{p}\right), \quad \forall l=\ldots,-1,0,1,2, \ldots .
$$

(ii) The pair $\left(\bar{\Phi}_{p}, \bar{\Gamma}_{p}\right)$ is not controllable if

$$
\operatorname{rank}\left(\sum_{k=0}^{p-1} S(k)\right)<m \text {. }
$$

For the proof (not included for length reasons), we will first investigate the structure of $\bar{\Phi}_{p}$ and $\bar{\Gamma}_{p}$. We then use the PBH eigenvector test to demonstrate the controllability of the system by defining some characteristic polynomials. If every actuator signal is updated during the sequence at least once, the result is that the system is proven to be controllable except when some specific sampling frequencies are used.

Remark 3. The conditions in (26) and (27), for the eigenvalues $\lambda$ of $\Phi(\tau)=e^{A \tau}$, can be regarded as an extended pathological sampling frequency condition for limited communication systems. The condition of (26) can be easily satisfied by appropriate selection of the sampling time.

Based on Theorem 1, we may introduce the idea of feasible control sequences.

Definition 6. A $p$-periodic sequence $\sigma$ will be called feasible if it generates a sequence of matrices $S$ such that

$$
\operatorname{rank}\left(\sum_{k=0}^{p-1} S(k)\right)=m \text {. }
$$

Condition (29) ensures that each actuator control signal is updated at least once during the control sequence.

Definition 7. A $p$-periodic sequence $\sigma$ will be called minimum feasible if the actuators are controlled only once in the sequence i.e. the sequence is feasible according to Definition 6 and $\sum_{k=0}^{p-1} m_{r}(k)=m$.

\footnotetext{
$\overline{2 \text { Note that condition (26) is void for } p-k_{f}^{(i)}}+\hat{q}\left(k_{f}^{(i)}\right) \leq 1$
}

From Definition 5 it follows that, for a minimum feasible sequence, $\bar{q}^{(i)}=k_{f}^{(i)} \Rightarrow \hat{q}^{(i)}\left(k_{f}^{(i)}\right)=k_{f}^{(i)}$. The condition on the eigenvalues, $\lambda$, for controllability with minimum feasible sequences reduces to

$$
\begin{gathered}
\lambda \neq \exp \left(\frac{2 \pi n \sqrt{-1}}{p}\right), \quad n=1,2, \ldots, p-1, \\
\lambda_{1} \neq \lambda_{2} \exp \left(\frac{2 \pi l \sqrt{-1}}{p}\right), \quad \forall l=\ldots,-1,0,1,2, \ldots,
\end{gathered}
$$

for any pair of eigenvalues $\left(\lambda_{1}, \lambda_{2}\right)$ of $\Phi$ and for $p>1$. The condition is strictly dependent on the period $p$. This applies to round-robin schedules where the control signal delay is in fact constant for all actuators.

Remark 4. The minimum feasible sequence provides a tight lower bound on the sequence length $p$ to preserve controllability of the NCS. By assuming $m_{r}=m_{r}(k)$, for all $k$, (i.e. the number of actuators controlled at any time is constant over the sequence) this lower bound is given by

$$
\beta_{l}=\left\lceil\frac{m}{m_{r}}\right\rceil,
$$

where $\lceil\cdot\rceil$ is the ceiling function.

If only one control signal is updated at any time tick (singlechannel case) then $\beta_{l}=m$.

\section{NCS WITHOUT ZOH}

In this section we extend the previous results to the same problem formulation as in Zhang and Hristu-Varsakelis [2006]. We consider a NCS where communication constraints apply to both input and output and no $\mathrm{ZOH}$ element is placed between the controller and the plant. The number of states of the augmented plant will not increase as the measurement and control signals are simply reset to zero when not updated. For the sensor and actuator scheduling, we need the following definition.

Definition 8. The sequence of input scheduling matrices

$$
S_{I}=\left\{S_{I}(0), S_{I}(1), \ldots, S_{I}(p-1)\right\}
$$

generated by the sequence

$$
\sigma_{I}=\left\{\sigma_{I}(0), \sigma_{I}(1), \ldots, \sigma_{I}(p-1)\right\}
$$

is defined as $S$ in Definition 2 and it determines the scheduling for the limited communication between controller output and plant input.

The sequence of output scheduling matrices

$$
S_{O}=\left\{S_{O}(0), S_{O}(1), \ldots, S_{O}(p-1)\right\},
$$

generated by the sequence

$$
\sigma_{O}=\left\{\sigma_{O}(0), \sigma_{O}(1), \ldots, \sigma_{O}(p-1)\right\}
$$

is defined in a similar way and it determines the scheduling for the limited communication for the plant output.

The sampled-data model with limited communication is

$$
\begin{aligned}
& x(j+1)=\Phi x(j)+\Gamma S_{I}(k) u(j), \quad x(0)=x_{0}, \\
& y(j)=S_{O}(k) C x(j), \quad k=\bmod (j, p),
\end{aligned}
$$

where $x(j), u(j), \Phi$ and $\Gamma$ are the same as in (3) and $y(j) \in \mathbb{R}^{p_{o}}$ are the limited plant outputs. $S_{I}(k)$ and $S_{O}(k)$ (Definition 8) are the scheduling matrices that, for simplicity, and without loss of generality, are assumed to have the same period $p$. We consider the multi-channel case where more than one control signal 
can be updated at any time tick. To eliminate the periodicity of (37), we let $j=p l+k$ and lift the linear time variant system (as described in Section 2.2) giving the linear time-invariant system

$$
\begin{aligned}
& x(p l+p)=\Phi^{p} x(p l)+\breve{\Gamma}_{p} \breve{u}(p l), \\
& \breve{y}(p l)=\breve{C}_{p} x(p l),
\end{aligned}
$$

where

$$
\begin{aligned}
& \breve{u}(p l)=\left[u(p l)^{T} u(p l+1)^{T} \cdots u(p l+p-1)^{T}\right]^{T}, \\
& \breve{y}(p l)=\left[y(p l)^{T} y(p l+1)^{T} \cdots y(p l+p-1)^{T}\right]^{T},
\end{aligned}
$$

and

$$
\begin{aligned}
& \breve{\Gamma}_{p}=\left[\begin{array}{l:l:l}
\Phi^{p-1} \Gamma S_{I}(0) & \Phi^{p-2} \Gamma S_{I}(1) & \cdots
\end{array} \Phi^{0} \Gamma S_{I}(p-1)\right], \\
& \breve{C}_{p}=\left[\begin{array}{c}
S_{O}(0) C \Phi^{0} \\
S_{O}(1) C \Phi \\
\vdots \\
S_{O}(p-1) C \Phi^{p-1}
\end{array}\right], \\
& \breve{\Gamma}_{p} \in \mathbb{R}^{m \times m p} \text { and } \breve{C}_{p} \in \mathbb{R}^{p_{o} p \times n} \text {. }
\end{aligned}
$$

\subsection{Controllability and Observability}

The following theorem gives conditions for the controllability of the system in (38).

Theorem 2. Suppose that the pair $(\Phi, \Gamma)(4)$ is controllable, $\Phi$ is invertible and $\operatorname{rank}(\Gamma)=m$. Moreover, all $m$ actuators are required for controllability of the plant, i.e. $\left(\Phi, \Gamma_{r}\right)$ is not controllable where $\Gamma_{r}$ is an input distribution matrix covering only part of the input range of $\Gamma$ (rank $\left(\Gamma_{r}\right)<m$ and $\left.\operatorname{rank}\left(\left[\Gamma_{r} \Gamma\right]\right)=m\right)$.

(i) The pair $\left(\Phi^{p}, \breve{\Gamma}\right)$ (the lifted system) is controllable if

$$
\operatorname{rank}\left(\sum_{k=0}^{p-1} S_{I}(k)\right)=m
$$

and for any pair of eigenvalues, $\left(\lambda_{1}, \lambda_{2}\right)$ of $\Phi$, the following holds:

$$
\lambda_{1} \neq \lambda_{2} \exp \left(\frac{2 \pi l \sqrt{-1}}{p}\right), \quad \forall l=\ldots,-1,0,1,2, \ldots
$$

(ii) The pair $\left(\Phi^{p}, \breve{\Gamma}\right)$ is not controllable if

$$
\operatorname{rank}\left(\sum_{k=0}^{p-1} S_{I}(k)\right)<m \text {. }
$$

\section{Proof See Appendix A.}

The following corollary gives conditions for the observability of the system in (38) using the concept of duality:

Corollary 1. Suppose that the pair $(C, \Phi)$ is observable, $\Phi$ is invertible and $\operatorname{rank}(C)=p_{o}$. Moreover, all $p_{o}$ sensors are required for observability of the plant, i.e. $\left(C_{r}, \Phi\right)$ is not observable where $C_{r}$ is an output distribution matrix covering only part of the output range of $C$ (rank $\left(C_{r}\right)<p_{o}$ and $\left.\operatorname{rank}\left(\left[C_{r}^{T} C^{T}\right]\right)=p_{o}\right)$.

(i) The pair $\left(\breve{C}_{p}, \Phi^{p}\right)$ (the lifted system) is observable if

$$
\operatorname{rank}\left(\sum_{k=0}^{p-1} S_{O}(k)\right)=p_{o}
$$

and for any pair of eigenvalues, $\left(\lambda_{1}, \lambda_{2}\right)$ of $\Phi$, the following holds:

$$
\lambda_{1} \neq \lambda_{2} \exp \left(\frac{2 \pi l \sqrt{-1}}{p}\right), \quad \forall l=\ldots,-1,0,1,2, \ldots
$$

(ii) The pair $\left(\breve{C}_{p}, \Phi^{p}\right)$ (the lifted system) is not observable if

$$
\operatorname{rank}\left(\sum_{k=0}^{p-1} S_{O}(k)\right)<p_{o}
$$

Remark 5. Note that $\Phi$ is always invertible because we are dealing with sampled-data systems. However, Theorem 1, 2 and Corollary 1 can be generalized to the analysis of any discrete system, $(C, \Phi, \Gamma)$, as long as $\Phi$ is invertible. This in particular applies to systems which are not derived from continuous-time systems through sampling as exemplified later.

Remark 6. The concept of feasible and minimum feasible sequences defined for $\sigma$ in Definition 6 and 7 also applies for $\sigma_{I}$ and $\sigma_{O}$. Hence, the $p$-periodic sequences $\sigma_{I}$ and $\sigma_{O}$ will be called feasible if they generate sequences of matrices $S_{I}$ and $S_{O}$ such that

$$
\operatorname{rank}\left(\sum_{k=0}^{p-1} S_{I}(k)\right)=m \text { and } \operatorname{rank}\left(\sum_{k=0}^{p-1} S_{O}(k)\right)=p_{o},
$$

and minimum feasible if the actuators or the sensor signals appear only once in the sequence.

Remark 7. For controllability and observability the strict lower bound on $p$ (see Remark 4) together with the proof of existence of a sequence at this length is an improvement to the results of Hristu-Varsakelis [2007, 2008], Zhang and Hristu-Varsakelis [2006], Ionete and Çela [2006] where sequences of length $\beta_{u}=$ $\left\lceil\frac{n}{m_{r}}\right\rceil n$ or $\beta_{u}=n,\left(\beta_{u} \geq \beta_{l}\right)$ are considered. The additional spectral condition on the system can be easily satisfied by appropriate selection of the sampling time.

Remark 8. The results on multirate systems by Colaneri et al. [1992] can be regarded as a special case of our NCS analysis. $\circ$

\section{EXAMPLES}

For comparative reasons, let us consider the numerical example in [Hristu-Varsakelis, 2007, Sec. IV A] where a 2-input, 2output, unstable plant is controlled through a shared communication medium with both sensor and actuator scheduling. The system is fully observable and stabilizable (but not fully controllable). Only one sensor and only one actuator can be $\mathrm{read} /$ controlled at any time tick. We ignore any delay. The original plant is stabilizable and detectable and it is shown by Hristu-Varsakelis [2007] that the shortest communication sequence that preserve stabilizability and detectability is $\sigma_{I}=$ $\sigma_{O}=\{2,2,1\}$ (3-periodic).

The system of [Hristu-Varsakelis, 2007, Sec. IV A] can be split into the uncontrollable stable subsystem and the controllable but partially unstable system. By analyzing the controllable subsystem via Theorem 2 and the whole system via Corollary 1 employing the definition of minimum feasible sequence (Definition 7) only a 2-periodic communication sequence (e.g. 
$\left.\sigma_{I}=\sigma_{O}=\{2,1\}\right)$ would have sufficed to preserve stabilizability and detectability. This is valid with and without $\mathrm{ZOH}$ strategy.

In the example of [Zhang and Hristu-Varsakelis, 2006, Sec. 5] the following discrete-time plant is considered

$$
\Phi=\left[\begin{array}{cc}
0 & -1 \\
1 & 0
\end{array}\right], \quad \Gamma=\left[\begin{array}{ll}
0 & 1 \\
1 & 0
\end{array}\right]
$$

and it is claimed by the authors that a round-robin communication sequence (i.e. $\sigma_{I}=\{1,2\}$ or $\sigma_{I}=\{2,1\}$ ) would fail to preserve the plant's controllability. The controllability preserving sequence calculated by the algorithms in Zhang and Hristu-Varsakelis [2006] is $\sigma_{I}=\{2,2,1\}$ (3-periodic). This is consistent with our results because of condition (42). In fact, the eigenvalues of $\Phi$ in (48) are

$$
\lambda_{1}=\sqrt{-1} \text { and } \lambda_{2}=-\sqrt{-1}
$$

and clearly

$$
\lambda_{1}=\lambda_{2} \exp \left(\frac{2 \pi l \sqrt{-1}}{2}\right), \quad l=\ldots,-3,-1,1,3, \ldots
$$

However, if the sequence is 3 -periodic

$$
\lambda_{1} \neq \lambda_{2} \exp \left(\frac{2 \pi l \sqrt{-1}}{3}\right), \quad \forall l=\ldots,-1,0,1,2, \ldots
$$

and therefore the system preserves controllability (of course, with condition (29) satisfied too). Notice that not all the actuators are required for controllability of the plant and consequently the trivial sequence $\sigma_{I}=\{1\}$ or $\sigma_{I}=\{2\}$ can also preserve controllability.

\section{CONCLUSION}

In this paper, we considered a class of linear NCSs subject to scheduled communication in the control loop. Using the lifting technique we proved that communication sequences that preserve controllability and observability of NCS can be shorter than previously established and we set a tight lower bound to it. In particular, we proved that a communication sequence that avoids particularly defined pathological sampling rates and updates each signal only once is sufficient to preserve controllability and observability. This result is valid with and without $\mathrm{ZOH}$ strategy. Suitable examples showed the advantage of our results over other work.

\section{Appendix A. PROOF OF THEOREM 2}

We will use the PBH eigenvector test to prove controllability of the lifted augmented system without $\mathrm{ZOH}$. The following auxiliary Lemma is required:

Lemma 1. If the pair $(\Phi, \Gamma)(4)$ is controllable and any pair of eigenvalues, $\left(\lambda_{1}, \lambda_{2}\right)$ of $\Phi$ satisfies (27)

(i) then the pair $\left(\Phi^{p}, \Gamma\right)(4)$ is controllable

(ii) the lefthand nonzero eigenvectors of $\Phi^{p}$ are also the nonzero eigenvectors, $v$, of $\Phi$ satisfying:

$$
v \Phi=\lambda v, \quad v \Phi^{p}=\lambda^{p} v, \quad v \Gamma \neq 0
$$

The Lemma is a direct consequence of Remark 1 and the proof of [Chen and Francis, 1995, Theorem 3.2.1]. (i). We need to prove that there is no left eigenvector of $\Phi^{p}$ that is orthogonal to $\breve{\Gamma}_{p}$ (see [Kailath, 1980, p. 135]). By Lemma 1 , the eigenvectors $v$ of $\Phi^{p}$ are given by the eigenvectors of $\Phi$ and $v \Gamma \neq 0$. Then (from (40))

$$
\begin{aligned}
& v \breve{\Gamma}_{p}=\left[\begin{array}{ll:lll}
v \Phi^{p-1} \Gamma S_{I}(0) & v \Phi^{p-2} \Gamma S_{I}(1) & \cdots & v \Phi^{0} \Gamma S_{I}(p-1)
\end{array}\right]
\end{aligned}
$$

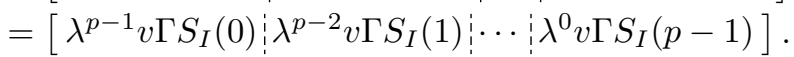

Note that $\Phi(\tau)=e^{A \tau} \neq 0$ and, for each eigenvalue $\lambda \neq 0$ of $\Phi$, the matrices $S_{I}(k)$ will be able to select every column on $\Gamma$ because $\operatorname{rank}\left(\sum_{k=0}^{p-1} S_{I}(k)\right)=\operatorname{rank}(\Gamma)=m$. By Lemma 1 , $\left(\Phi^{p}, \Gamma\right)$ is controllable and $v \Gamma \neq 0$, which implies $v \breve{\Gamma}_{p} \neq 0$.

(ii). If the sequence does not select every column of $\Gamma$ then $v \Gamma \neq 0$ is not satisfied and it will not be possible to guarantee $v \breve{\Gamma}_{p} \neq 0$.

\section{REFERENCES}

M. Ben Gaid, A. Çela, and Y. Hamam. Optimal integrated control and scheduling of networked control systems with communication constraints: application to a car suspension system. IEEE Trans. on Control Systems Technology, 14(4):776-787, 2006.

R. Brockett. Stabilization of motor networks. In Proc. of the 34th IEEE Conf. on Decision and Control, volume 2, pages 1484-1488, 1995.

T. Chen and B. Francis. Optimal Sampled-data Control Systems. Springer, London, 1995.

P. Colaneri, R. Scattolini, and N. Schiavoni. LQG optimal control of multirate sampled-data systems. IEEE Trans. on Automatic Control, 37(5):675-682, 1992.

F. Göktas, J. Smith, and R. Bajcsy. Telerobotics over communication networks. In Proc. of the IEEE Conf. on Decision and Control, volume 3, pages 23992404, 1997.

G. Gwaltney and J. Briscoe. Comparison of communication architectures for spacecraft modular avionics systems. Technical report, NASA/TM-2006214431, 2006.

D. Hristu-Varsakelis. On the period of communication policies for networked control systems, and the question of zero-order holding. In Proc. of the $46^{\text {th }}$ IEEE Conf. on Decision and Control, pages 38-43, 2007.

D. Hristu-Varsakelis. Short-period communication and the role of zero-order holding in networked control systems. IEEE Trans. on Automatic Control, 53(15):1285-1290, 2008.

C. Ionete and A. Çela. Structural properties and stabilization of NCS with medium access constraints. In Proc. of the $45^{\text {th }}$ IEEE Conf. on Decision and Control, pages 1141-1146, 2006.

T. Kailath. Linear Systems. Prentice-Hall, 1980.

M. Kimura. Preservation of stabilizability of a continuous time-invariant linear system after discretization. Int. J. Systems Sci., 21(1):65-92, 1990.

H. Kopetz. Event-triggered versus time-triggered real time system. Technical Report 8/91, Inst. für Technische Informatik, Technische Universität Wien, 1991.

G. Leen and D. Heffernan. Expanding automotive electronic systems. IEEE Computer, 35(1):88-93, 2002.

F Lian, J. Moyne, and D. Tilbury. Implementation of networked machine tools in reconfigurable manufacturing systems. In Proc. of the 2000 Japan-USA Symposium on Flexible Automation, Ann Arbor, MI, 2000.

M. Oda, T. Doi, and K. Wakata. Tele-manipulation of a satellite mounted robot by an on-ground astronout. In Proc. of the IEEE Int. Conf. on Robotics \& Automation, pages 1891-1896, 2001.

H. Rehbinder and M. Sanfridson. Scheduling of a limited communication channel for optimal control. Automatica, 40(3):491-500, 2004.

G. Walsh and H. Ye. Scheduling of networked control systems. IEEE Control Systems Magazine, 21:57-65, 2001.

L. Zhang and D. Hristu-Varsakelis. Communication and control co-design for networked control systems. Automatica, 42(6):953-958, 2006. 


\title{
Cranfield University
}

\section{CERES https://dspace.lib.cranfield.ac.uk}

2009-06-18

\section{Controllability, Observability in Networked Control}

\author{
Longo, Stefano
}

\section{Elsevier}

Stefano Longo, Guido Herrmann and Phil Barber. Controllability, Observability in Networked

Control, IFAC Proceedings Volumes, Volume 42, Issue 6, 2009, Pages 295-300

https://doi.org/10.3182/20090616-3-IL-2002.00051

Downloaded from Cranfield Library Services E-Repository 